their analysis and resist the temptation of saying 'doctor knows best'.

First, were the index assessments made at the same time? Mental distress fluctuates and therefore one assessment may not concur with another. Second, the findings that 24 of the 33 cases subsequently received in-patient care informally suggests that many of the recommendations were at least partly unnecessary because informal admission subsequently followed.

Third, the emergence of dangerous behaviour in the undetained group seemed little different to the detained group, suggesting that hospital detention may not be the most effective way to treat the disturbed behaviour.

Clearly statements like an individual "lacks insight" and that "electro-convulsive therapy is not necessary" make a nonsense of the purpose of mental health legislation but whether these sorts of disagreements should be aired so publicly seems questionable.

We have recently started to undertake multidisciplinary (clinical) audit to look at untoward incidents, and at meetings such as these the various ideological differences can be addressed. A paper of this sort could, I am sure, be presented by social workers to illustrate how autocratic psychiatrists are and that we need more liberal mental health legislation with clients having more say. Mental Health Act assessments need to be handled carefully and a decision reached only after considered discussion with all interested parties.

A. N. WEAR, Royal South Hants Hospital, Southampton SO9 4PE

\section{Medical care of the long-term mentally ill in hostels}

Sir: Dr Essex's review (Psychiatric Bulletin, 1992. 16, 126) concerning different patterns of care for long-term psychiatric patients living in hostels (Horder, 1990) needs re-addressing. One hostel, where a GP shared the care with a psychiatrist, is a model fraught with potential problems. Both professionals visit the hostel which encourages continuing institutionalisation, the residents continue to receive care passively and the potential for duplication of work exists. At another hostel, the psychiatrist dealt with all the problems which smacks of a need to over-control ex-hospital patients or an inability to let go. Who is dependent on whom? Why were the residents registered with a GP? This is poor use of a psychiatrist's time. A third hostel had the residents consulting GPs as ordinary NHS patients, and consulting a psychiatrist when necessary (similar to long-stay rehabilitation hostels in Gloucester). This model has not led to problems in communication or defining medical responsi- bility in Gloucester (cf. Horder, 1990) and hostel staff can access the psychiatrist and help patients see their GP.

Dr Essex's suggestion that only GPs are necessary, and will not have to deal with unusual problems, cannot be true. He misleads by saying the hostels contained chronic schizophrenic patients. Over one third of patients have a different psychiatric diagnosis. Some have multiple diagnoses encompassing organic and functional psychiatry as well as physical morbidity. Hence the need for shared care. Many also have severe communication and behavioural problems.

Dr Essex wonders whether GPs should be given extra remuneration for the responsibility of these hostel patients. Using the third hostel model negates any justification for this - local GPs do not complain of extra workload and some feel it is reduced.

Dr Essex has not mentioned that Dr Horder's study found "the GPs in the enquiry were unanimous in saying that the work had not been unduly difficult or arduous, and that off-duty work was minimal". Several made spontaneous comments about its interest and value.

The debate about medical and psychiatric care and responsibility for the long-term mentally ill in the community continues. Further informative studies like Dr Horder's should be welcomed as the move out of the asylums will necessitate caring for more ex-hospital patients in these settings.

HORDER, E. (1990) Medical Care in Three Psychlatric Hostels, Hampstead and District Health Authortty. Hampstead and South Barnet GP Forum and Hampstead Department of Community Medicine.

JES RoBerts, Coney Hill Hospital, Coney Hill, Gloucester GLA 7QJ

\section{The mental health needs of refugee children}

Sir: The Department of Health's Health Care (Medical) Division has been in negotiation with the Refugee Council over the possibility of forming a list of those mental health professionals who have an interest in, or are engaged in, work with refugee children in Britain.

I would like to hear from any psychiatrists, even if we have had previous contact, who would be willing to have their names put on such a list, with a contact address and telephone number and the nationality(ies) of the children they could help. This list would be held by Ms Ros Finlay of the Refugee Council and will include clinical psychologists and child psychotherapists as well.

Donald J. BROOKSBANK, Department of Health, Wellington House, 133-155 Waterloo Road, London SE1 8UG 